\title{
Klotho enhances Fox03-mediated manganese superoxide dismutase expression by negatively regulating PI3KJAKT pathway during tacrolimus-induced oxidative stress
}

\author{
Sun Woo Lim ${ }^{1,2}$, Long Jin ${ }^{1,2}$, Kang Luo ${ }^{1,2}$, Jian Jin ${ }^{1,2}$, Yoo Jin Shin ${ }^{1,2}$, Sung Yi Hong ${ }^{2}$ and Chul Woo Yang ${ }^{*, 1,2,3}$
}

\begin{abstract}
Mammalian members of the forkhead box protein 0 (FoxO) class of transcription factors are implicated in the regulation of oxidative stress, and FoxO proteins are negatively regulated by the phosphatidylinositol 3-kinase (PI3K)-AKT signaling pathway. We examined the effect of Klotho on the PI3K/AKT pathway and manganese superoxide dismutase (MnSOD) during tacrolimus (Tac)-induced oxidative stress. Klotho-treated mice showed decreased Tac-induced oxidative stress accompanied by functional and histological improvements. Klotho inhibited the PI3K/AKT-mediated phosphorylation of FoxO3a and enhanced FoxO3a binding to the MnSOD promoter. Klotho increased MnSOD mRNA and protein expression in mitochondria. In addition, Klotho reduced Tac-induced mitochondrial dysfunction and decreased mitochondrial reactive oxygen species production, and these effects were enhanced by blocking PI3K activity with LY294002. Collectively, our data showed that Klotho protects Tac-induced oxidative stress by negatively regulating the PI3K/AKT pathway and subsequently enhancing FoxO3a-mediated MnSOD expression.
\end{abstract}

Cell Death and Disease (2017) 8, e2972; doi:10.1038/cddis.2017.365; published online 3 August 2017

Klotho, an anti-aging protein, is predominantly expressed in the brain and kidneys, ${ }^{1}$ it extends the mouse lifespan by $20-30 \%,{ }^{2}$ and Klotho-deficient mice show multiple agerelated phenotypes and experience premature death. ${ }^{1,3}$ Importantly, recent data showed an association between human longevity and a functional variant of Klotho. ${ }^{4} \mathrm{~A}$ recent report showed that Klotho overexpression in mice extended the lifespan by repressing of insulin or insulin-like growth factor-1 signaling, an evolutionarily conserved mechanism for

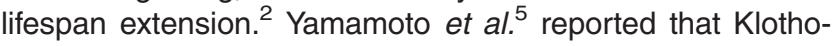
induced expression of the manganese superoxide dismutase (MnSOD) protein, a mitochondrial antioxidant enzyme that detoxifies superoxides, in COS, HELA, and $\mathrm{CHO}$ cells and that the anti-oxidative effect of Klotho potentially contributes to its anti-aging properties. Findings related to the roles of Klotho in renal function have shown that Klotho-gene expression levels are significantly decreased in the kidneys of patients with chronic renal failure ${ }^{6}$ and ischemia-reperfusion injury. ${ }^{7}$ Klotho overexpression can preserve renal function by suppressing the development of renal failure ${ }^{7}$ and glomerulonephritisinduced cellular senescence. ${ }^{8}$ However, the regulatory mechanism of Klotho in renal disease remains poorly understood.

Forkhead box protein $\mathrm{O}$ (FoxO) transcription factors regulate various downstream target genes, including those involved in cellular differentiation, growth, survival, the cell cycle, glucose and lipid metabolism, stress, and reactive oxygen species (ROS) detoxification. ${ }^{9}$ The phosphatidylinositol 3-kinase (PI3K)-Akt serine-threonine kinase (AKT) signaling pathway regulates FoxO through phosphorylation. The AKT-mediated phosphorylation of FoxO inhibits FoxO activity by promoting its interaction with 14-3-3 proteins and nuclear exportation, and also by inducing its proteasomal degradation. ${ }^{10}$ FoxO3a can upregulate MnSOD expression. ${ }^{2,5,11}$ Thus, FoxO3a functions as a negative regulator of mitochondrial ROS production, ${ }^{12}$ and thereby closely associates with resistance to oxidative stress.

Calcineurin inhibitor (CNI)-based regimens are the most popular immunosuppressive drugs used for solid organ transplantation, and 2 CNIs (cyclosporine (CsA) and tacrolimus (Tac)) are available in clinical practice. ${ }^{13}$ In $\mathrm{T}$ cells, CsA bind to cyclophilin and Tac binds to FKBP12. ${ }^{14-17}$ CsA-cyclophilin and Tac-FKBP12 complexes can bind to calcineurin, resulting in inhibition of the phosphatase activity of calcineurin. This inhibition then impairs translocation of the nuclear factor of activated T cells, ${ }^{18-20}$ which regulates IL-2 transcription and thus T-cell activation. $^{21-23}$ Despite the specific inhibition of T-cell activation, long-term treatment with CNIs causes serious adverse effects, and nephrotoxicity is a major issue in solid organ transplantation. The pathogenesis of $\mathrm{CNI}$-induced nephrotoxicity is still undetermined, but oxidative stress caused by ROS is regarded as a common pathway of CNI-induced renal injury. ${ }^{24}$

\footnotetext{
${ }^{1}$ Transplant Research Center, Seoul St. Mary's Hospital, College of Medicine, The Catholic University of Korea, Seoul, Korea; ${ }^{2}$ Institute for Convergent Research Consortium for Immunologic Disease, Seoul St. Mary's Hospital, College of Medicine, The Catholic University of Korea, Seoul, Korea and ${ }^{3}$ Division of Nephrology, Department of Internal Medicine, Seoul St. Mary's Hospital, College of Medicine, The Catholic University of Korea, Seoul, Korea

${ }^{*}$ Corresponding author: CW Yang, Division of Nephrology, Department of Internal Medicine, Seoul St. Mary's Hospital, College of Medicine, The Catholic University of Korea, Seoul, Korea. Tel: +82 2225868 51; Fax: +82 2225868 17; E-mail: yangch@ catholic.ac.kr

Received 23.1.17; revised 04.7.17; accepted 05.7.17; Edited by C Muñoz-Pinedo
} 
We previously demonstrated that $\mathrm{CNI}$-induced nephropathy was improved by combined treatment with an anti-oxidative agent such as statin, angiotensin II blockade, or $\mathrm{N}$-acetylcysteine. ${ }^{25-28}$ We also found a causal relationship between Klotho expression and the renoprotective effects of antioxidant agents. Using Klotho $+/-$ mice, we found that Klotho deficiency renders the kidney more susceptible to Tac-induced injury, which was closely associated with aggravated Tac-induced oxidative stress and reduced MnSOD and nuclear FoxO expression. ${ }^{29}$ The current study focused on whether Tac-induced oxidative stress could be inhibited by exogenous treatment with the Klotho protein, which can reduces Tac-induced oxidative stress by FoxO3a-mediated MnSOD expression via negative regulating of the PI3K/AKT pathway.

\section{Results}

Klotho ameliorates Tac-induced nephrotoxicity. Table 1 shows changes in the functional parameters in the experimental group after Tac and recombinant Klotho (rKlotho) treatment for 4 weeks. The Tac+rKlotho group showed a lower level of water intake, urine volume, and serum creatinine (Scr) than did the Tac group. Immunoreactivity against renal kidney injury marker-1 (KIM-1) and tubulointerstitial fibrosis (Figure 1) in tissue sections was reduced by Tac treatment in the Tac+rKlotho group.

Effect of Klotho administration on Tac-induced oxidative stress and apoptosis in mice. Figure 2 shows immunohistochemical staining results for 8-hydroxy-2'deoxyguanosine (8-OHdG; Figures 2a-d) and 4-hydroxy-hexenal (4-HHE; Figures $2 \mathrm{e}-\mathrm{h}$ ), and 24-h urinary excretion of $8-\mathrm{OHdG}$ (Figure $2 \mathrm{~m}$ ), a marker of oxidative DNA or lipid damage. Immunoreactivity against $8-\mathrm{OHdG}$ and $4-\mathrm{HHE}$, and the urine 8-OHdG concentration markedly increased in the Tac group, which was reversed by rKlotho treatment. We also evaluated whether Klotho protects against Tac-induced apoptosis, which is an important cell death mechanism in Tac-induced nephrotoxicity. The number of terminal deoxynucleotidyl transferase dUTP nick end labeling (TUNEL)-positive cells in tissue sections was significantly higher in the Tac group versus the vehicle $(\mathrm{VH})$ group, and this was reduced by the addition of rKlotho (Figures $2 \mathrm{i}-\mathrm{I}, \mathrm{p}$ ).

Effect of Klotho administration on Tac-induced mitochondrial ultrastructure and MnSOD expression in mice. The results shown in Figure 3 implied that rKlotho may regulate mitochondrial function, which is strongly associated with ROS production and apoptosis. Therefore, we studied morphology changes in mouse mitochondria by electron microscopy. Ultrastructural analysis revealed that Tac treatment caused a reduction in the number, area, and size of mitochondria, and rKlotho administration overcame those changes (Figures $3 a-d, i-k)$. We also found that Tac treatment decreased immunoreactivity against MnSOD

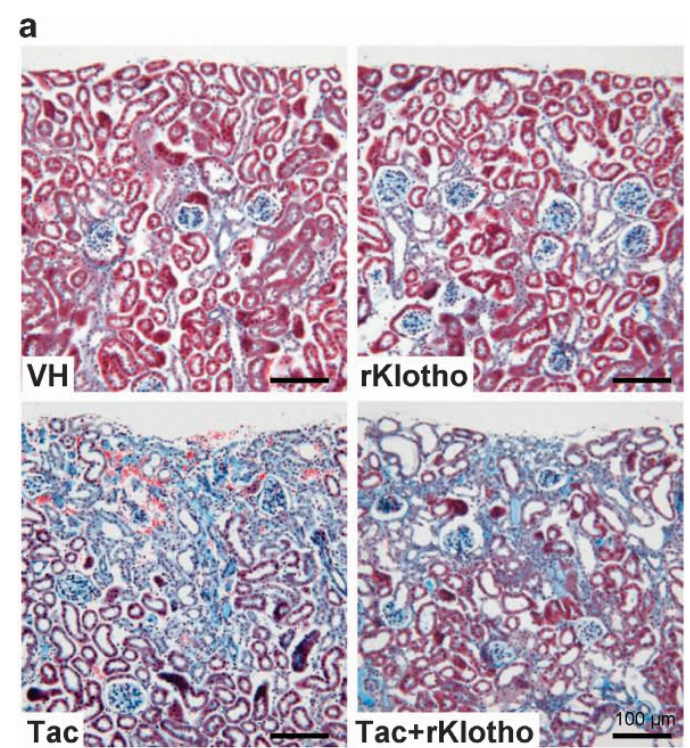

b

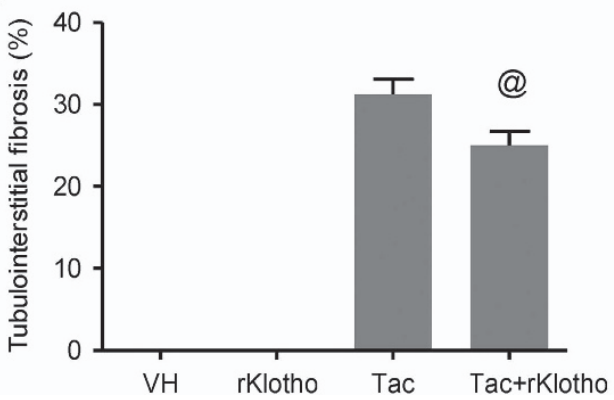

Figure 1 Effect of rKlotho administration on Tac-induced tubulointerstitial fibrosis in an experimental mouse model. ( $\mathbf{a}$ and $\mathbf{b}$ ) Histological analysis in the renal cortex in mice treated with Tac for 4 weeks showed striped tubulointerstitial fibrosis, mononuclear cell infiltration, and tubular atrophy. rKlotho treatment significantly reduced these damages compared with Tac treatment. Scale bar $=100 \mu \mathrm{m}$. The data are presented as the mean \pm S.E. ${ }^{\circledR} P<0.05$ versus the Tac group

Table 1 Effect of administration of rKlotho on Tac-induced renal injury in mice

\begin{tabular}{lcrr}
\hline & VH & rKlotho & Tac+rKlotho \\
\hline Body weight (g) & $31 \pm 1$ & $32 \pm 1$ & $27 \pm 1^{1}$ \\
Water intake (ml/day) & $2.2 \pm 0.14$ & $2.3 \pm 0.19$ & $25 \pm 1$ \\
Urine volume (ml/day) & $0.3 \pm 0.03$ & $0.4 \pm 0.02$ & $0.67^{1,2}$ \\
Scr (mg/dl) & $0.15 \pm 0.2$ & $0.8 \pm 0.11^{1,2}$ & $0.4 \pm 0.3^{1,2}$ \\
KIM-1 (\%) & $10.9 \pm 2.0$ & $0.15 \pm 0.2$ & $0.33^{3}$ \\
& & $9.0 \pm 3.4$ & $0.10^{3}$ \\
\end{tabular}

Abbreviations: KIM-1, kidney injury marker-1; rKlotho, recombinant Klotho; Scr, serum creatinine; Tac, tacrolimus; VH, vehicle

The values shown are the mean $\pm \mathrm{S}$.E. $(n=8){ }^{1} \mathrm{P}<0.05$ versus $\mathrm{VH} ;{ }^{2} \mathrm{P}<0.05$ versus rKlotho; ${ }^{3} \mathrm{P}<0.05$ versus Tac. 

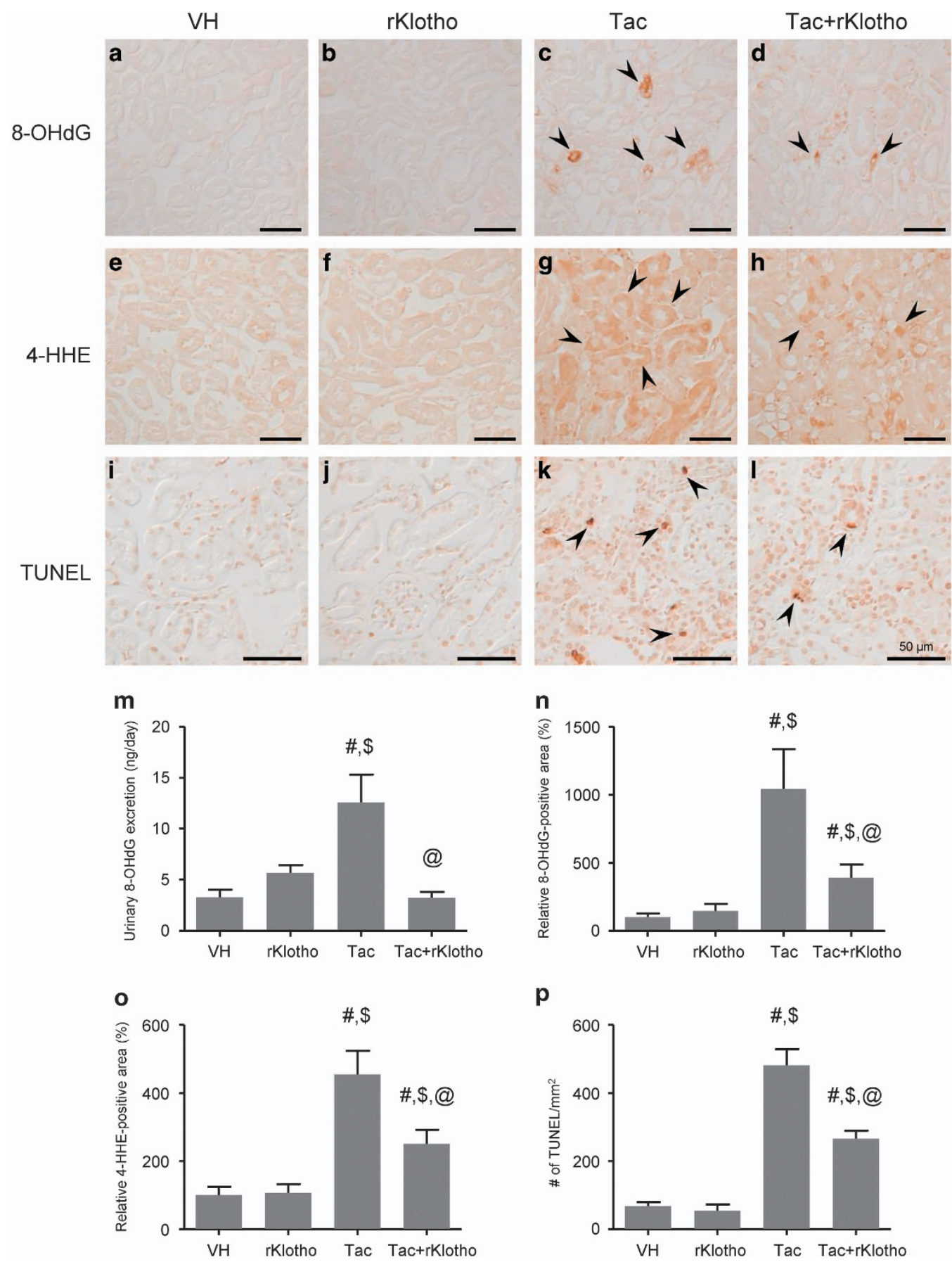

p

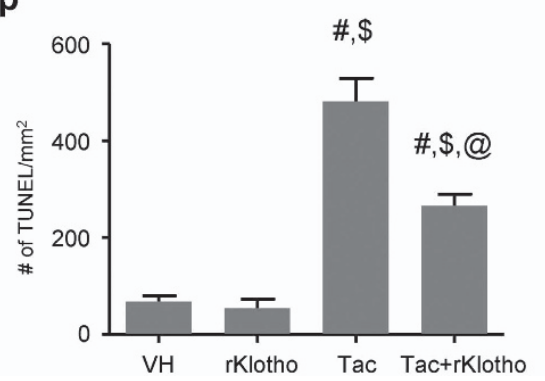

Figure 2 Effect of rKlotho administration on Tac-induced oxidative stress and apoptosis in an experimental mouse model. Representative images and quantification of immunohistochemistry for 8-OHdG (a-d and $\mathbf{n}$ ) and 4-HHE (e-h and $\mathbf{o}$ ) and TUNEL assays ( $\mathbf{i}-\mathbf{I}$ and $\mathbf{p})$, using tissue sections from mouse kidneys. High immunoreactivity was significantly decreased by the co-administration of rKlotho. (m) Urinary 8-OHdG excretion per day. Tac-induced 8-OHdG excretion was lowered by rKlotho co-administration. The arrows indicate 8-OHdG, 4-HHE, and TUNEL-positives. Scale bar $=50 \mu \mathrm{m}$. The data are presented as the mean $\pm \mathrm{S} . \mathrm{E}$. ${ }^{\sharp} P<0.05$ versus the VH group; ${ }^{\$} P<0.05$ versus the rKlotho groups; ${ }^{\circledR} P<0.05$ versus the Tac group

(green fluorescence), which was mainly localized in the mitochondria of tubular cells. This effect was also significantly inhibited by rKlotho (Figures $3 e-h, I)$.

Klotho induces MnSOD expression by regulating the PI3K/AKT/FoxO3a pathway during Tac- treatment. To determine whether rKlotho upregulates the MnSOD signaling pathway, we performed immunofluorescence for p-AKT and $\mathrm{t}$-FoxO3a in an experimental mouse kidney section. Confocal microscopy revealed that Tac treatment increased $p$-AKT expression in proximal tubule, and rKlotho co-treatment reduced this increase (Figures $4 a-d$ ). Intense immunofluorescent staining of t-FoxO3a in the cytoplasm was detected in the proximal tubules of the Tac group (Figures $4 \mathrm{e}-\mathrm{g}$ ). 

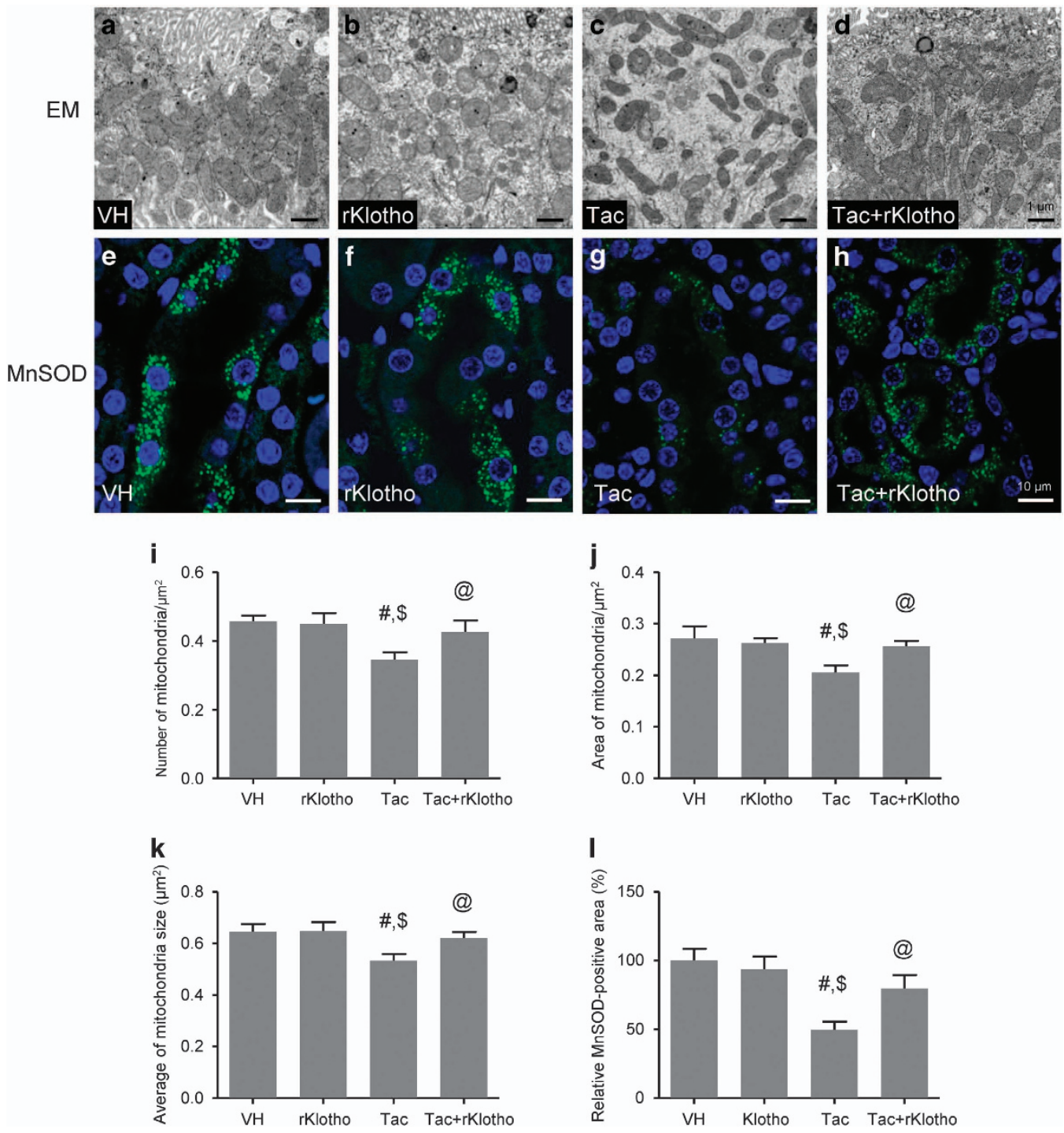

Figure 3 Effect of rKlotho administration on Tac-induced mitochondrial ultrastructure and MnSOD expression in an experimental mouse model. Representative transmission electron microscopy images of the mitochondrial ultrastructure in proximal tubules (a-d) and its quantification in a mouse kidney (i-k). MnSOD immunofluorescence (green fluorescence) (e-h) and its quantification (I) in each group. The Tac groups showed mitochondrial disorders such as a lower number, area, and size of mitochondria, and rKlotho administration reversed those changes. Note that the reduction and intensity of MnSOD-positive dots was also improved by rKlotho administration. Scale bars $=1 \mu \mathrm{m}(\mathrm{a}-\mathrm{d})$ or $10 \mu \mathrm{m}(\mathrm{e}-\mathrm{h})$. The data are presented as the mean \pm S.E. ${ }^{\#} P<0.05$ versus the VH group; ${ }^{\$} P<0.05$ versus the rKlotho groups; ${ }^{\circledR} P<0.05$ versus the Tac group

Interestingly, t-FoxO3a was also located in the nucleus in the rKlotho co-treated group (Figure 4h). mRNA levels of MnSOD were detected by in situ hybridization in tissue sections, and Tac treatment markedly reduced the mRNA level, whereas rKlotho treatment recovered the intensity. Using the HK-2 proximal tubule cell line, we further evaluated whether rKlotho upregulates MnSOD expression via the PI3K/AKT/FoxO3a pathway. We also examined cell viability with or without the PI3K-specific inhibitor LY294002 during Tac and rKlotho treatment in HK-2 cells. The protective effect of rKlotho further increased after LY294002 treatment (Figure 5a). Immunoblot analysis using whole-cell lysates revealed that Tac treatment activated PI3K/AKT, thereby increasing FoxO3a phosphorylation (causing deactivation or cytoplasmic retention) and decreased MnSOD expression (Figure 5b). Similarly, reduction of the MnSOD level by Tac treatment was also increased with rKlotho treatment in protein samples of the mitochondrial fraction.

Next, we confirmed that Klotho-induced FoxO3a nuclear translocalization by immunostaining for total FoxO3a (t-FoxO3a) expression. t-FoxO3a mainly localized to the cytoplasm in the Tac group, but nuclear t-FoxO3a immunoreactivity occurred after Klotho treatment. (Figure 5c). We studied t-FoxO3a immunoreactivity in the LY294002-treated group as a positive control doe nuclear staining. Furthermore, a chromatin immunoprecipitation (ChIP) assay revealed that rKlotho treatment increased FoxO3a binding to the MnSOD promoter and the MnSOD mRNA level (Figures $6 a$ and $b$ ).

Klotho reduces Tac-induced mitochondrial ROS production and mitochondrial dysfunction. Next, we evaluated whether Klotho-dependent MnSOD induction via the 


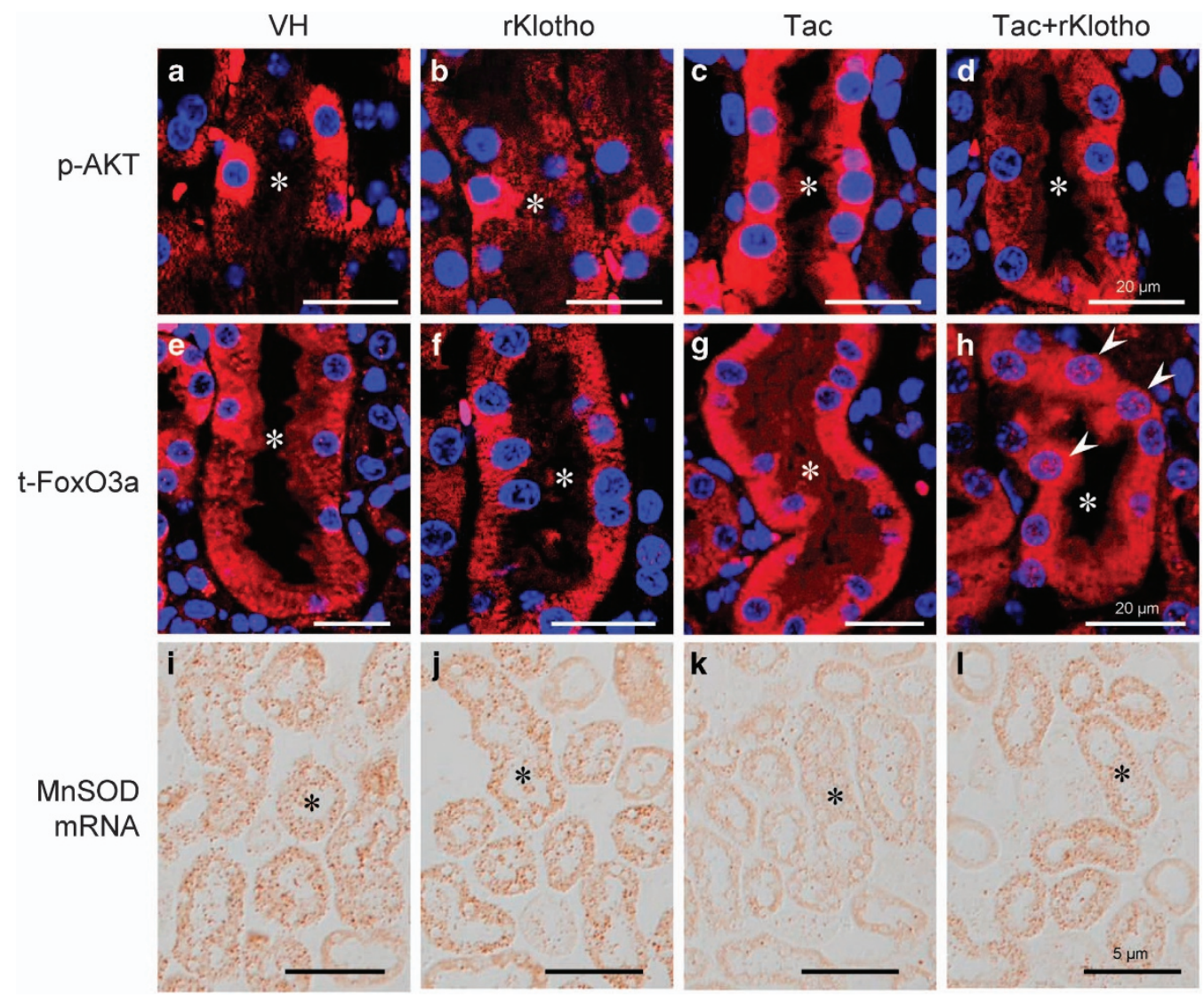

Figure 4 Klotho-induced MnSOD mRNA expression by regulating the PI3K/AKT/FoxO3a pathway in an experimental mouse model. Representative immunofluorescence images of phosphorylated AKT (p-AKT, a-d) and total FoxO3a (t-FoxO3a, e-h), and in situ hybridization of MnSOD mRNA (i-l) in tissue sections from mouse kidneys. Arrowheads point to nuclear punctate expression of t-FoxO3a in the Tac+rKlotho group. Asterisks indicate proximal tubules. Scale bars $=20 \mu \mathrm{m}(\mathrm{a}-\mathrm{h})$ or $5 \mu \mathrm{m}(\mathrm{i}-\mathrm{I})$

$\mathrm{PI3K} / \mathrm{AKT} / \mathrm{FoxO} 3 \mathrm{a}$ pathway regulation decreased Tacinduced mitochondrial ROS accumulation. MitoSOX Red staining for mitochondrial superoxide $\left(\mathrm{O}^{-}\right)$along with confocal microscopy and flow cytometric analysis in HK-2 cells revealed that rKlotho significantly decreased Tac-induced MitoSOX Red fluorescence, and suppression of PI3K by LY294002 further reduced its expression (Figures $7 \mathrm{a}-\mathrm{C}$ ). We also found that MitoTracker fluorescence significantly decreased in the Tac group and the addition of rKlotho significantly the fluorescence, demonstrating that rKlotho reduced mitochondrial damage (Figures $7 \mathrm{~d}$ and e). In addition, the significantly decreased number of polarized cells (JC-1 red) in the Tac group was reduced by rKlotho treatment. In contrast, the increase in depolarized cells was reduced in the Tac+rKlotho group (Figure 8). The oxygenconsumption rate (OCR) of cells was assessed, as shown in Figure 9. rKlotho-treated cells showed a higher OCR than cells treated with Tac alone (Figure 9a). Compared with Tac alone-treated cells, Tac+rKlotho-treated cells showed significantly higher basal respiration, ATP-linked respiration, maximal respiration, and spare respiratory capacity. These data suggested that rKlotho may protect against Tac-induced mitochondrial dysfunction (Figures 9b-e). Blocking of PI3K by LY294002 during Tac+rKlotho treatment further increased mitochondrial function.
Klotho reduces Tac-induced apoptosis in HK-2 cells. To examine whether rKlotho inhibits mitochondrial pathways associated with apoptosis, we performed flow cytometric analysis by annexin $\mathrm{V}$ staining and immunoblotting against pro- or anti-apoptotic genes. The higher percent of annexin V-positive cells after Tac treatment was significantly reduced by rKlotho treatment and even more so by LY294002 (Figures 10a and b). Using whole-cell lysates, we found that Tac-dependent $\mathrm{Bcl}-2$ downregulation was reversed by rKlotho. Pro-apoptotic markers (Bax, activated caspase-9 and -3 ) were markedly increased by Tac treatment, and this phenomenon was blocked in rKlotho-treated cells. PI3K inhibition with LY294002 further improved the anti-apoptotic effect of rKlotho (Figure 10c).

\section{Discussion}

The results of present study clearly demonstrated that Klotho inhibited PI3K/AKT-mediated phosphorylation of FoxO3a and subsequent enhancement of FoxO3a binding to the MnSOD promoter. Indeed, Klotho increased MnSOD mRNA and protein expression in mitochondria during Tac-induced toxicity in HK-2 cells. These findings suggest that Klotho increases resistance to Tac-induced oxidative stress in the kidneys via negative regulation of the PI3K/AKT pathway and that 
a

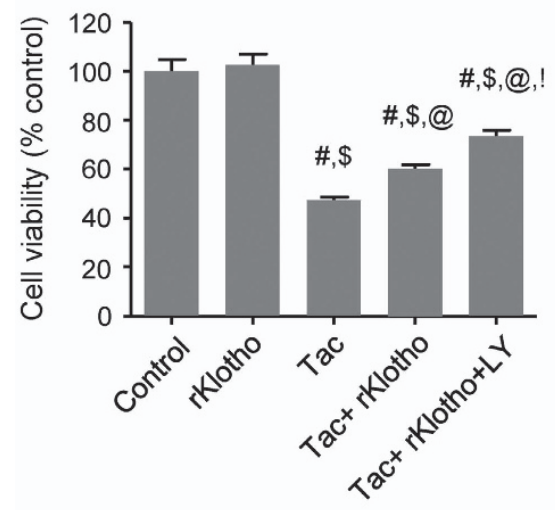

b

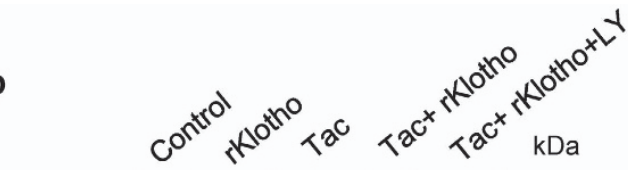

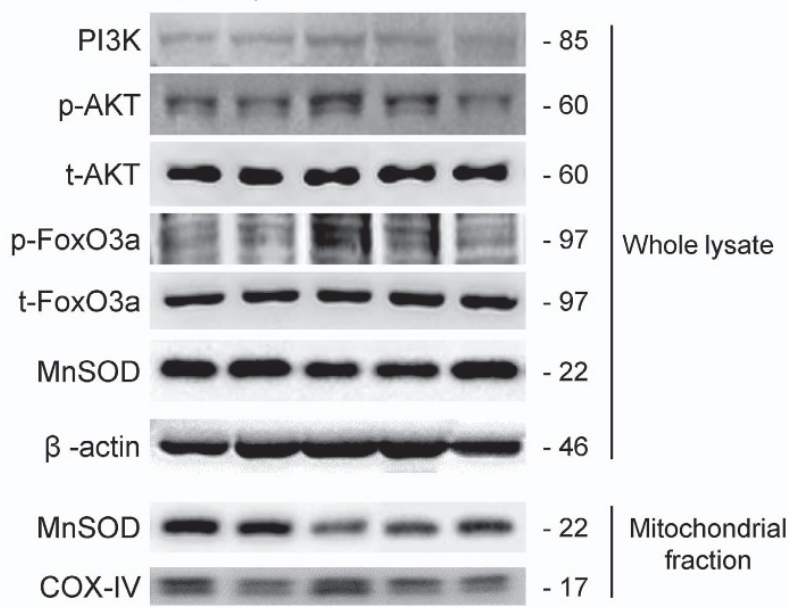

c Control Tac

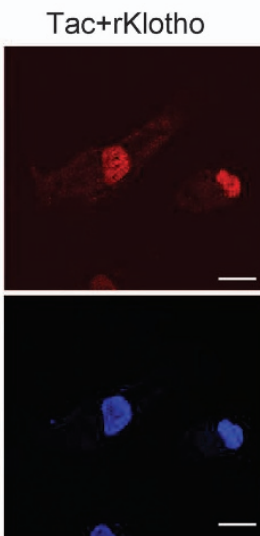

Tac+rKlotho+LY
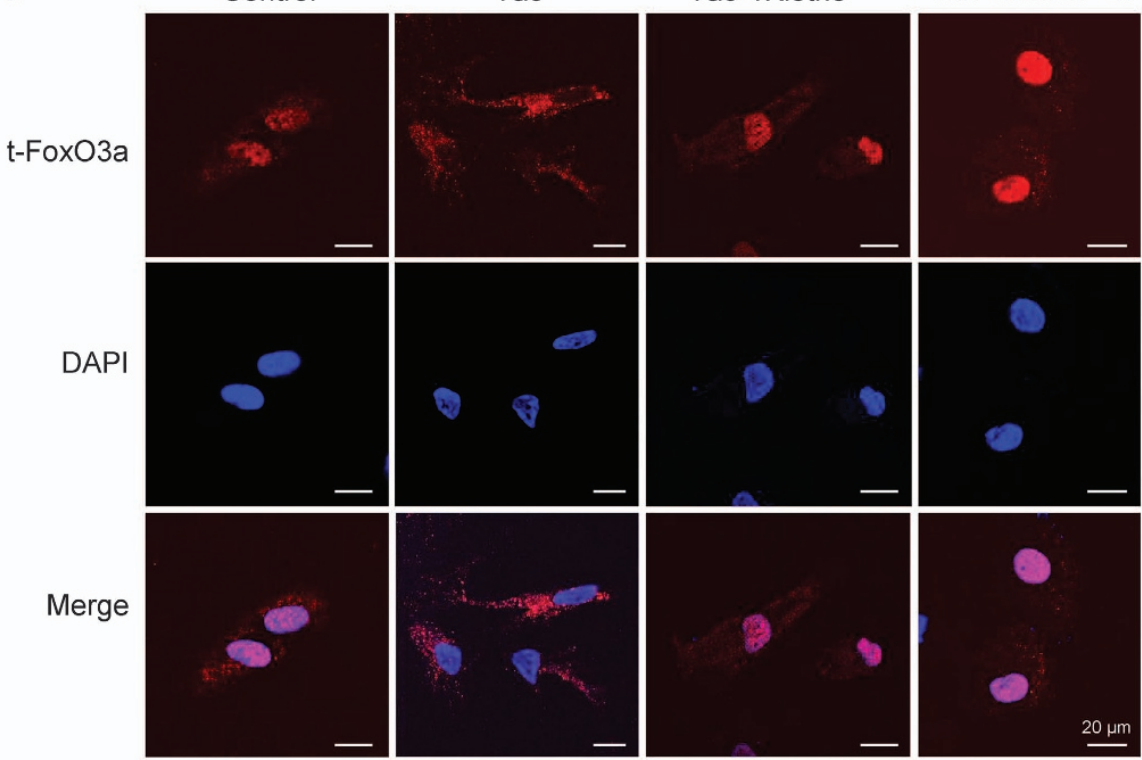

Figure 5 Klotho induces MnSOD expression by regulating the PI3K/AKT/FoxO3a pathway in Tac-treated HK-2 cells. HK-2 cells were seeded in culture plates at $90 \%$ confluence. On the next day, the cells were treated with Tac $(60 \mu \mathrm{g} / \mathrm{ml})$ in the absence or presence of $1 \mu \mathrm{g} / \mathrm{ml} \mathrm{rKlotho}$ and $25 \mu \mathrm{M}$ LY294002 (LY, PI3K inhibitor) for $12 \mathrm{~h}$. Before the end of the treatment, CCK-8 solution was added to each well for $2 \mathrm{~h}$ to measure the cell viability. (a) Cell-viability assays for the experimental group. (b) Whole-cell lysates and mitochondrial fractions were collected after each 12- $\mathrm{h}$ drug treatment to measure the protein expression of PI3K, phosphorylated AKT (p-AKT), phosphorylated FoxO3a (p-FoxO3a), and MnSOD. All proteins were normalized to $\beta$-actin or total (t) protein controls (for the whole-cell lysate) and cytochrome $\mathrm{c}$ oxidase subunit 4 (COX-IV) (for the mitochondrial fraction). (c) After each 12-h drug treatment, the cells were fixed with fixative and then immunofluorescence was performed with an antibody against t-FoxO3a. Nuclear translocation of t-FoxO3a was observed by confocal microscopy. Scale bar $=20 \mu \mathrm{m}$. The data are presented as the mean \pm S.E. ${ }^{\#} P<0.05$ versus the VH group; ${ }^{\$} P<0.05$ versus the rKlotho groups; ${ }^{\circledR} P<0.05$ versus the Tac group; ' $P<0.05$ versus the Tac+rKlotho group

FoxO3a-mediated MnSOD expression is involved in this process.

We previously demonstrated that Tac-induced nephropathy caused downregulation of renal Klotho, and drugs with antioxidant potential such as statin, angiotensin II blockade, and $\mathrm{N}$-acetylcysteine ${ }^{25-28}$ increased Klotho expression. This finding suggests a close association between Klotho and oxidative stress in Tac-induced renal injury, which was confirmed by showing that Klotho $+/$ - mice showed more
Tac-induced renal injury than wild-type littermates. ${ }^{29}$ In this study, we determined whether exogenous treatment of Klotho protein protects against Tac-induced oxidative injury. Co-treatment with the Klotho protein in mice improved renal function and attenuated tubulointerstitial fibrosis and renal KIM-1 expression, compared with Tac treatment alone. Next, we evaluated whether Klotho treatment decreases Tac-induced oxidative stress and apoptosis, and found that Klotho treatment dramatically downregulated oxidative stress 
a

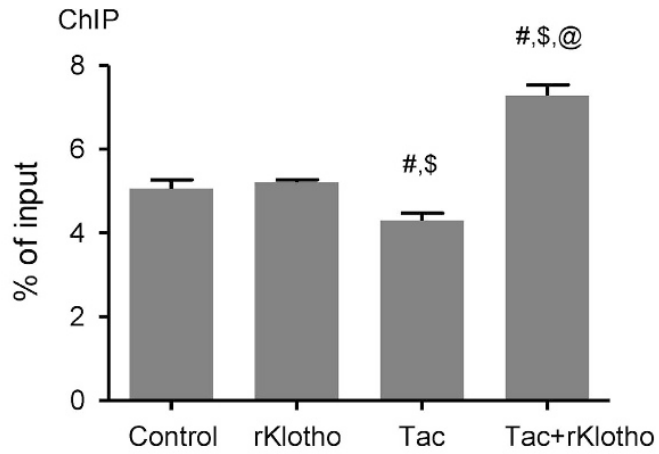

b

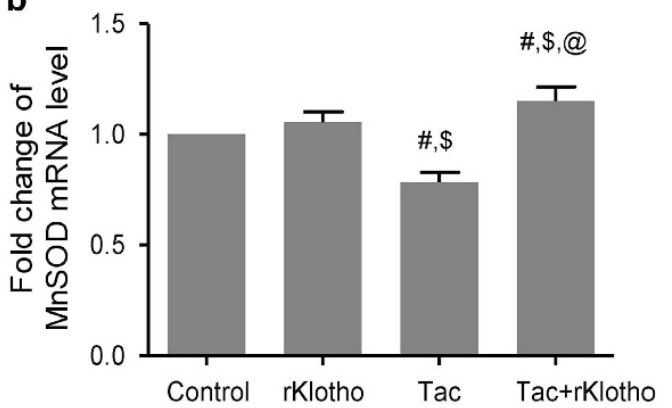

Figure 6 Klotho induces FoxO3a binding to the MnSOD promoter in Tac-treated HK-2 cells. HK-2 cells were seeded in culture plates at $90 \%$ confluence. On the next day, the cells were treated with Tac $(60 \mu \mathrm{g} / \mathrm{ml})$ in the absence or presence of $1 \mu \mathrm{g} / \mathrm{ml}$ rKlotho. After $12 \mathrm{~h}$, the cells were harvested to detect MnSOD transcription activity after nuclear FoxO3a translocation using a ChIP assay and quantitative real-time PCR (q-PCR) for MnSOD expression. (a) The FoxO3a protein/MnSOD promoter complex was analyzed by q-PCR with primer pairs for the MnSOD promoter region containing a FoxO3a-binding element. (b) MnSOD mRNA was detected by q-PCR in whole-cell lysates. Relative MnSOD expression was presented after normalization to cyclophilin A expression. The data are presented as the mean \pm S.E. ${ }^{\#} P<0.05$ versus the control group; ${ }^{\$} P<0.05$ versus the rKlotho groups; ${ }^{\circledR} P<0.05$ versus the Tac group

markers (8-OHdG and 4-HHE) and reduced the number of TUNEL-positive cells. These findings suggest that Klotho treatment may be beneficial in reducing structural and functional kidney impairment during Tac treatment.

We focused on mitochondrial ROS and MnSOD, based on previous reports that mitochondria are the primary cellular source of ROS and that MnSOD is as an important antioxidant enzyme in the mitochondria. ${ }^{30}$ Regarding the upregulation of MnSOD by Klotho, multiple mechanisms may contribute to protein kinase C-, PI3K/AKT-, and MAP kinase-mediated pathways. In the present study, we explored whether Klotho influences the PI3K/AKT pathway because downstream targets of the PI3K/AKT signaling pathway include those resulting from FoxO3a-mediated MnSOD expression. ${ }^{5,31}$ In kidney tissue sections, Klotho co-treatment reduced detection of phosphorylated AKT and enhanced nuclear FoxO3a expression. Reduced mRNA and protein levels of MnSOD (evaluated by in situ hybridization and immunofluorescence, respectively) were also observed following Klotho co-treatment (Figure 4). This finding suggests an association between the PI3K/AKT/FoxO-signaling pathway and MnSOD, and these relations might contribute to the anti-oxidative properties of Klotho against Tac-induced oxidative injury.
Next, we studied causal relationships between the PI3K/ AKT pathway and Klotho activity on in vitro study. In vitro study showed that co-treatment with rKlotho and Tac promoted nuclear FoxO3a translocation. Negative regulation of $\mathrm{PI3K} /$ AKT was confirmed with by treatment of with the LY294002, PI3K inhibitor. ChIP assays revealed that Klotho increased FoxO3a binding to the native MnSOD gene promoter, which was associated with increased MnSOD mRNA and protein expression. These results demonstrated that Klotho inhibited the Tac-induced PI3K/AKT pathway, thereby promoting translocation to the nucleus, which occurs is stabilized via direct binding of FoxO3a to the MnSOD promoter.

Next, we examined whether Klotho-induced MnSOD expression in mitochondria helped improved Tac-induced mitochondrial dysfunction mediated by inhibiting the PI3K/ AKT pathway. Mitochondria function (assessed by the OCR) showed that Klotho-treated cells had significantly higher basal respiration, ATP-linked respiration, maximal respiration, and spare respiratory capacity. In addition, mitochondrial membrane depolarization and excessive mitochondrial ROS formation during Tac treatment were also recovered by Klotho treatment. These protective effects of Klotho in mitochondria were related with to PI3K/AKT pathway by blocking PI3K with LY294002. In addition, we found that mitochondrial-associated apoptosis during Tac treatment was decreased by Klotho treatment via $\mathrm{Bcl}-2$ upregulation. These data suggested that the addition of Klotho protects against Tac-induced mitochondria dysfunction and apoptosis by negatively regulating the PI3k/AKT pathway.

Our study had several limitations. First, it remains unclear whether the ability of Klotho to confer oxidative stress resistance is entirely dependent on MnSOD induction, although this activity may be crucial for the anti-oxidative properties of Klotho. Overexpression of catalase removed ROS (catalase) in mitochondria and extended the lifespan of mice. ${ }^{32-34}$ A peroxiredoxin/thioredoxin-associated mechanism was associated with Klotho-induced ROS removal following brain injury. ${ }^{35}$ Second, we cannot explain clearly why Klotho treatment did not reverse weight loss in Tac-treated mice. However, we speculate that the protective effects of Klotho were not enough to promote weight recovery under these experimental conditions. Additional experiments are needed to resolve this problem in further investigations.

The proposed mechanism underlying the protective effect of Klotho in Tac-induced renal injury is summarized in Figure 11. Tac regulates PI3K/AKT-mediated phosphorylation of FoxO3a, which suggests that FoxO3a may be retained in the cytoplasm in an inactive form. However, Klotho promoted the nuclear export of FoxO3a by inhibiting PI3K/AKT activity and increasing MnSOD mRNA and protein expression. Through this mechanism, Klotho may protect against Tac-induced oxidative damage and apoptotic cell death. Finally, our results suggested that the Klotho protein or Klotho-enhancing compounds may provide treatment options for nephrotoxicity in the future.

Materials and Methods

Ethics statement. All procedures were performed in strict accordance with the recommendations of the ethical guidelines for animal studies. All experimental animal care protocols were approved by the Animal Care and Use Committee of the 
a
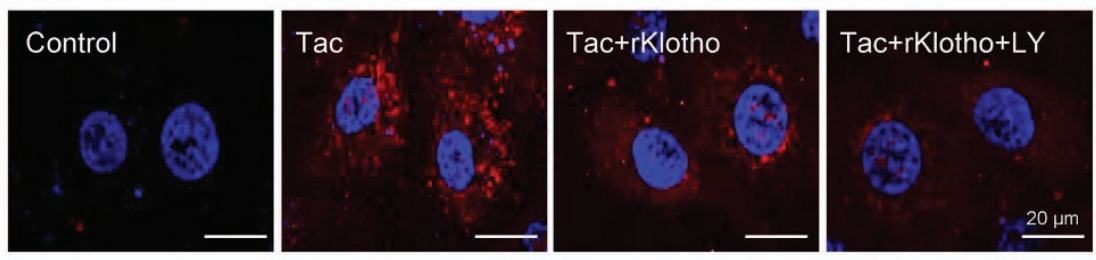

$b$
ल
ल

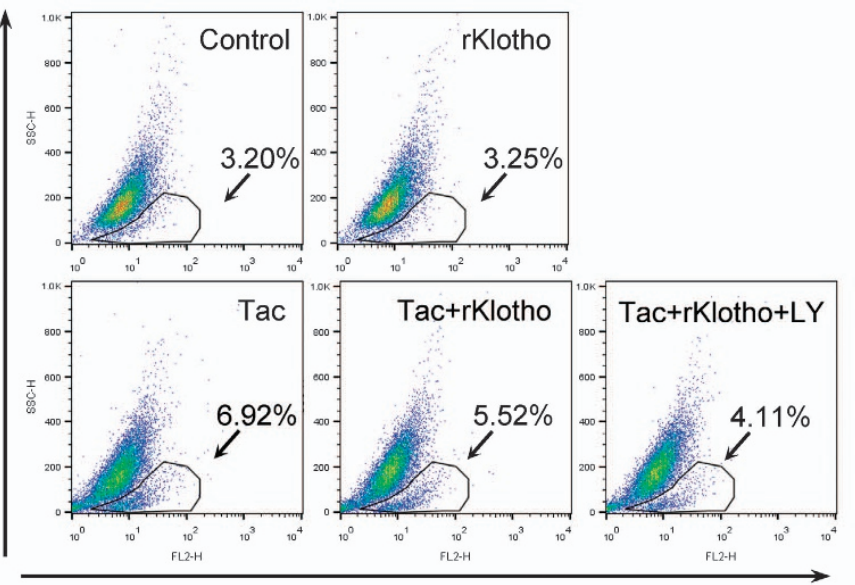

MitoSOX fluorescence

d
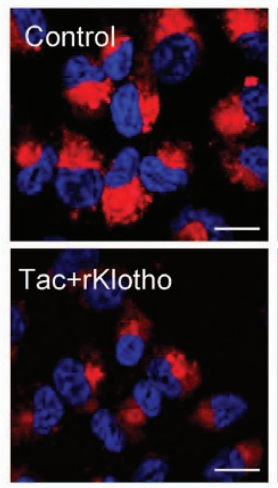

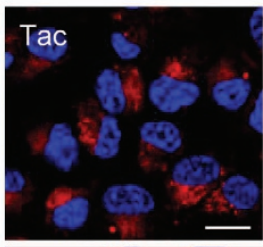

Tac+rKlotho+tY

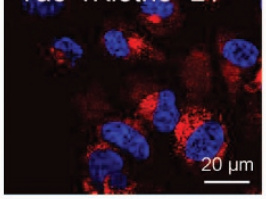

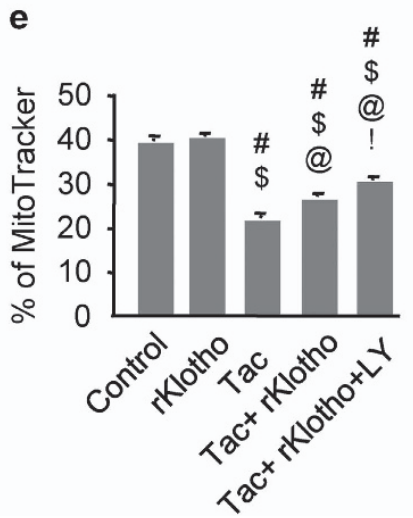

Figure 7 Klotho reduces Tac-induced mitochondrial ROS production and mitochondrial damage in HK-2 cells. HK-2 cells were seeded in culture plate at $90 \%$ confluence. On the next day, the cells were treated with Tac $(60 \mu \mathrm{g} / \mathrm{ml})$ in the absence or presence of $1 \mu \mathrm{g} / \mathrm{ml} \mathrm{rKlotho} \mathrm{and} 25 \mu \mathrm{M} \mathrm{LY} 294002$ (LY, PI3K inhibitor) for $12 \mathrm{~h}$. The cells were exposed in MitoSOX or MitoTracker, then analyzed by flow cytometry and confocal microscopy. MitoSOX staining to detect mitochondrial superoxide anion (O2-) levels by confocal microscopy (a) or flow cytometric analysis (b and $\mathbf{c}$ ). MitoTracker staining to detect the number of mitochondria by confocal microscopy (d). (e) Relative fluorescence intensity of MitoTracker staining by flow cytometry. LY; LY294002. Scale bar $=20 \mu \mathrm{m}$. The data are presented as the mean \pm S.E. ${ }^{\#} P<0.05$ versus the VH group; ${ }^{\$} P<0.05$ versus the rKlotho groups; ${ }^{\circledR} P<0.05$ versus the Tac group; $! P<0.05$ versus the Tac+rKlotho group

Catholic University of Korea (CUMC-2013-0056-02). Animals were killed under xylazine/rompun anesthesia, and every effort was made to minimize animal suffering.

Tac-induced renal injury mouse model. Eight-week-old male BALB/c mice (Orient Bio, Seongnam-Si, Korea) were housed with a 12-h/12-h light/dark cycle, a $0.01 \%$ salt diet (Research Diets, New Brunswick, NJ, USA), and water ad libitum. After acclimation for 1 week, weight-matched mice were randomized to four groups ( $n=8 /$ group) and treated together subcutaneously with $1.5 \mathrm{mg} /(\mathrm{kg}$ day) Tac (Prograft; Astellas Pharma, Ibaraki, Japan) or $10 \mathrm{ml} /(\mathrm{kg}$ day) VH (olive oil; SigmaAldrich, St. Louis, MO, USA), with or without recombinant mouse Klotho (rKlotho; $10 \mu \mathrm{g} / \mathrm{kg}$ once every 2 days, intraperitoneal injection; R\&D System, Minneapolis, MN, USA) for 4 weeks. The rKlotho used was the shed, circulating form of Klotho, not the transmembrane or secreted form. Administration routes and drug doses were chosen based on previous studies. ${ }^{2,29}$ After the 4-week treatment, animals were housed individually in metabolic cages (Tecniplast, Buguggiate, Italy) to measure urine volumes over $24 \mathrm{~h}$. Animals were then anaesthetized, and blood samples and tissue specimens were obtained for further analysis.

Creatinine measurements. Scr was measured using a quantitative enzyme colorimetric method (Stanbio Laboratory, Boerne, TX, USA), according to the manufacturer's instructions.

Renal fibrosis. Renal histological assessment was defined as the development of tubulointerstitial fibrosis using Trichrome-stained tissue sections. ${ }^{36}$ The extent of fibrosis was estimated in minimum of 20 fields per section by counting the percentage of injured area per field using the polygon program (TDI Scope Eye Version 3.6 for Windows; Seoul, Korea). Histopathological analysis was performed in randomly selected cortical fields of sections by a pathologist blinded to the identity of the treatment groups. 
a
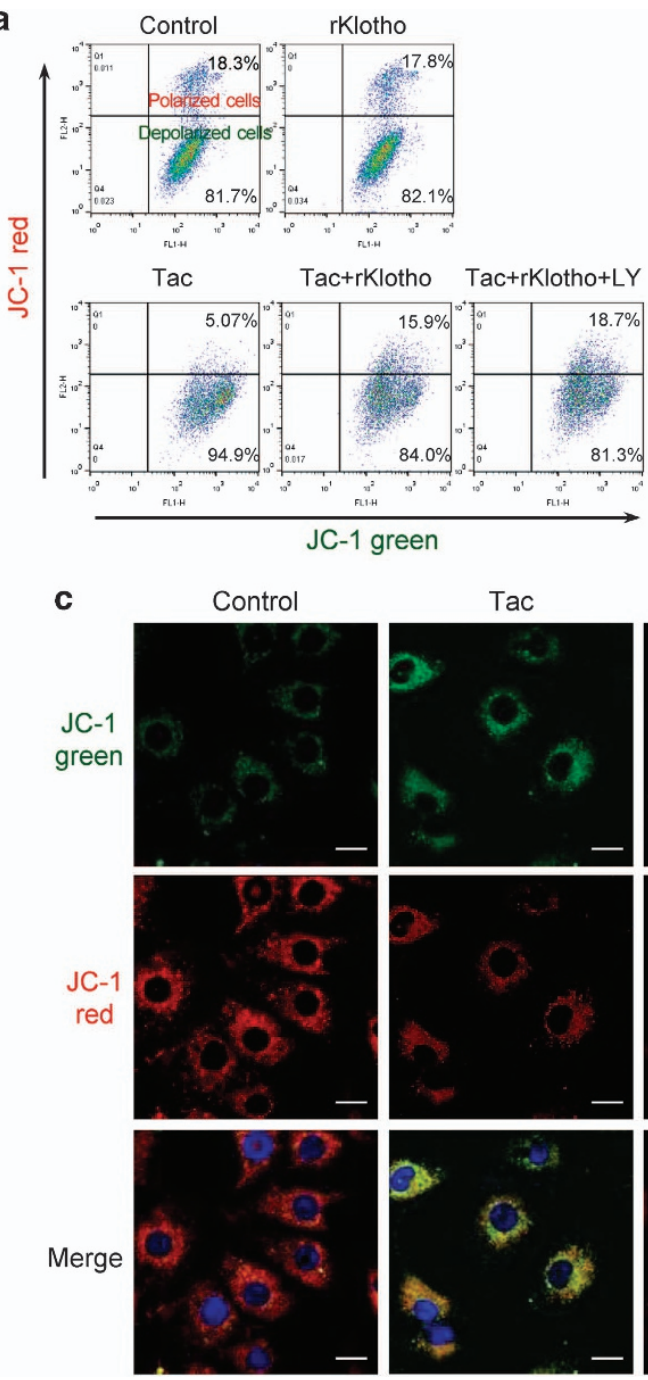

b

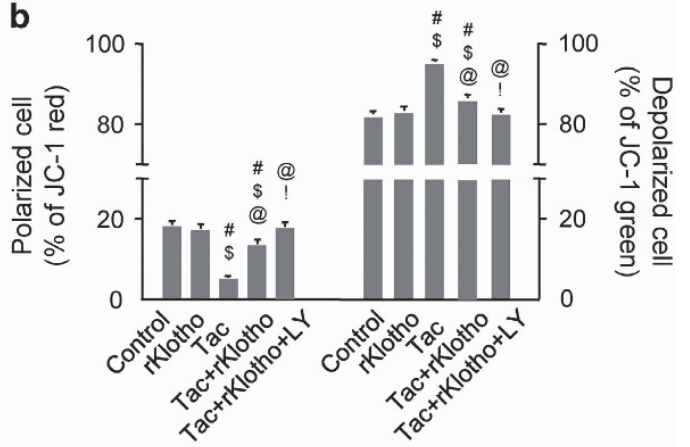

Tac+rKlotho

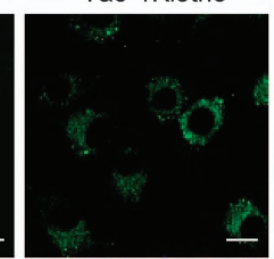

Tac+rKlotho+LY
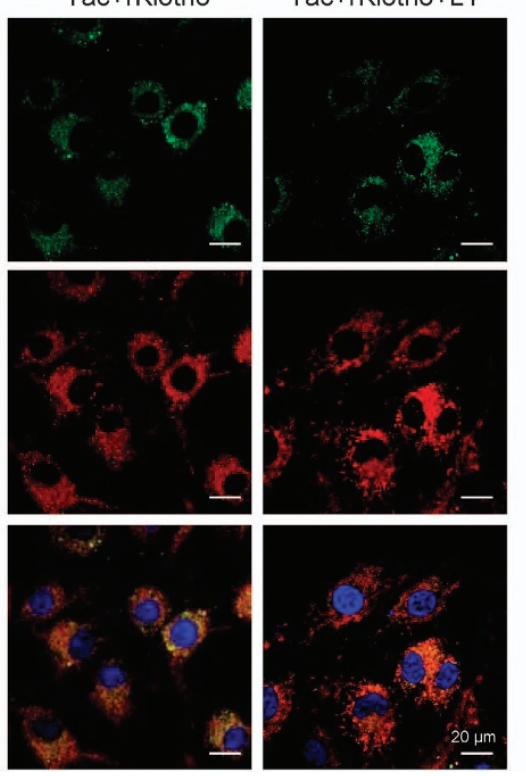

Figure 8 Klotho improves the mitochondrial membrane potential in Tac-treated HK-2 cells. HK-2 cells were seeded in culture plates at $90 \%$ confluence. On the next day, the cells were treated with Tac $(60 \mu \mathrm{g} / \mathrm{ml}$ ) in the absence or presence of $1 \mu \mathrm{g} / \mathrm{ml}$ rKlotho and $25 \mu \mathrm{M} \mathrm{LY} 294002$ (LY, PI3K inhibitor) for $12 \mathrm{~h}$. The cells were labeled with JC-1 to evaluate the mitochondrial membrane potential $(\Delta \Psi \mathrm{m})$ and then analyzed by flow cytometry and confocal microscopy. (a and $\mathbf{b})$ Flow cytometry plots and a quantitative graph for JC-1 labeling. (c) Confocal microscopy images for JC-1 staining. LY; LY294002. Scale bar $=20 \mu \mathrm{m}$. The data are presented as the mean \pm S.E. ${ }^{\#} P<0.05$ versus the VH group; ${ }^{\$} P<0.05$ versus the rKlotho groups; ${ }^{\circledR} P<0.05$ versus the Tac group; $! P<0.05$ versus the Tac+rKlotho group

Antibodies. The following primary antibodies were used for immunoblot analysis or immunohistochemical staining: anti-KIM-1 (ab56015; Abcam, Cambridge, UK), anti-8-OHdG (MOG-100 P; JalCA, Shizuoka, Japan), anti-4-HHE (MHH-030n; JalCA), anti-MnSOD (ab16953; Abcam), anti-PI3K (610045; BD Transduction Laboratories, San Jose, CA, USA), anti-p-AKT (Ser473) (9271 S; Cell Signaling Technology, Danvers, MA, USA), anti-t-AKT (9272 S; Cell Signaling Technology), anti-p-FoxO3a (9466 S; Cell Signaling Technology), anti-t-FoxO3a (2497 S; Cell Signaling Technology), anti- $\beta$-actin (A5441; Sigma-Aldrich), anti-COX-IV (A301899 A; Bethyl Laboratories, Montgomery, TX, USA), anti-Bcl-2 (sc-492; Santa Cruz Biotechnology, Santa Cruz, CA, USA), anti-Bax (DB005; Delta Biolabs, Gilroy, CA, USA), anti-active caspase-9 (9505 S; Cell Signaling Technology), and anti-active caspase-3 (AB3623; Millipore Corporation, St. Charles, MO, USA).

Immunohistochemical staining. Dewaxed sections were incubated in retrieval solution (pH 6.0), methanolic $\mathrm{H}_{2} \mathrm{O} 2$, and $0.5 \%$ Triton $\mathrm{X}-100$ and then washed in phosphate-buffered saline. Nonspecific binding sites were blocked in $10 \%$ normal donkey serum (Jackson ImmunoResearch, West Grove, PA, USA). Sections were incubated overnight at $4^{\circ} \mathrm{C}$ with primary antibodies and then with peroxidase- or Alexa Fluor 488 or Cyanine 3-conjugated secondary antibodies
(Molecular Probes, Carlsbad, CA, USA, Jackson ImmunoResearch) for $2 \mathrm{~h}$ at room temperature (RT). Peroxidase activity was detected using 3,3'-diaminobenzidine (DAB; Vector Laboratories, Burlingame, CA, USA) as a chromogen. For confocal images, the tissue sections were stained with 4',6-diamidino-2-phenylindole (Vector Laboratories). Stained tissues were viewed using a Zeiss LSM700 confocal microscope (Carl Zeiss Microscopy, GmbH, Jena, Germany). Quantification was performed inof $\sim 20$ randomly selected areas was performed per for each animal in each group. Quantitative analysis was performed by calculating the percent positive area showing the same intensity, using histogram equalization (TDI Scope Eye).

TUNEL staining. Apoptotic cells in tissue sections were detected by the TUNEL method and staining with the In Situ Apoptosis Detection Kit (Millipore Corp.). TUNEL-positive cells were counted in $\sim 20$ randomly selected nonoverlapping areas per animal in each group.

In situ hybridization. Visualization of MnSOD mRNA transcripts in tissues was done according to the supplier instructions for the RNAScope 2.5 HD Detection Kit (322371; Advanced Cell Diagnostics, Hayward, CA, USA) using a validated probe for mouse MnSOD (439361; Advanced Cell Diagnostics). In brief, $5 \mu \mathrm{m}$ 
a
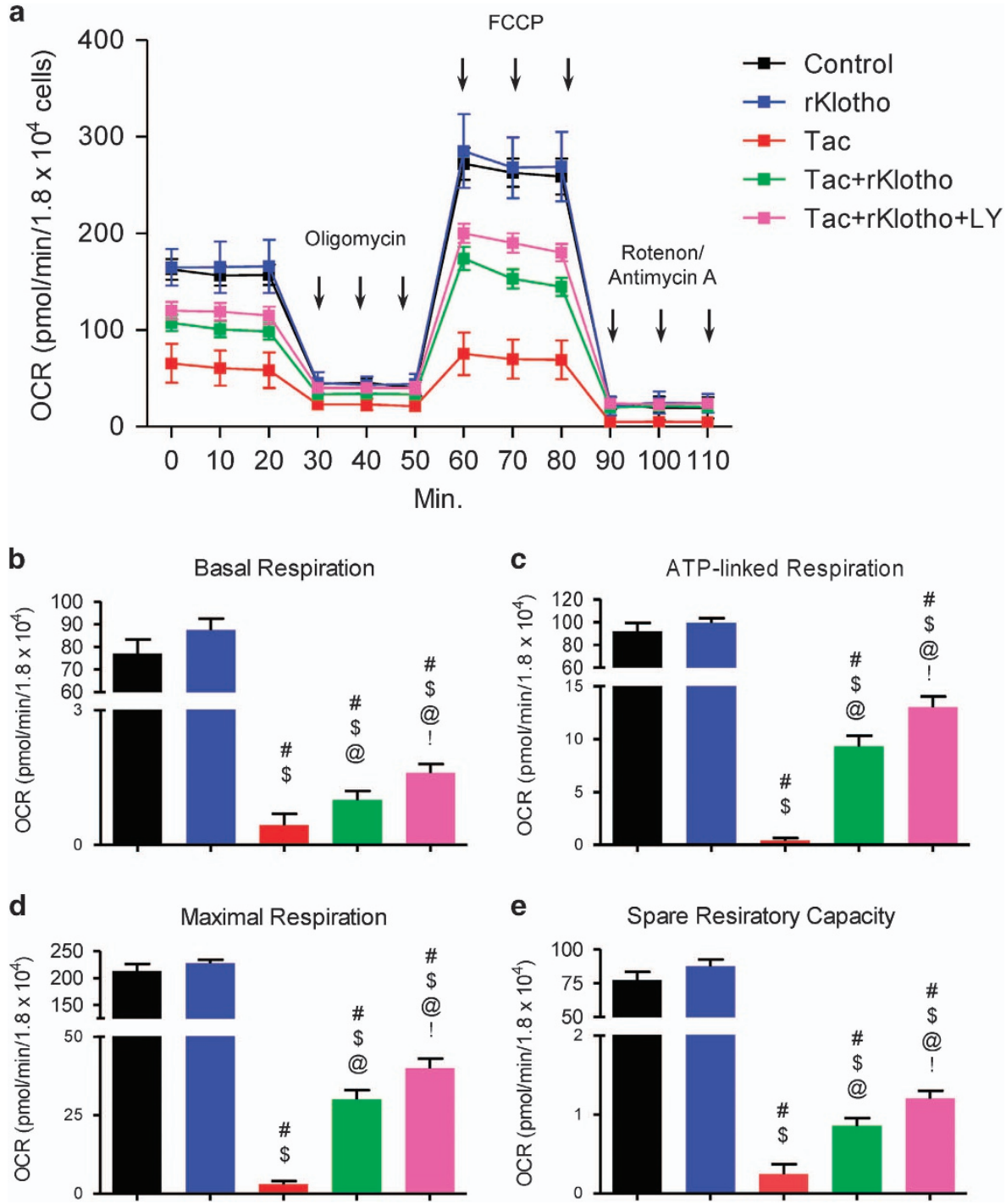

e

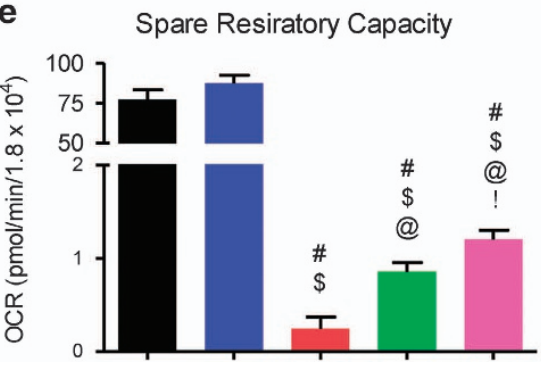

Figure 9 Klotho reduces Tac-induced mitochondrial dysfunction in HK-2 cells. HK-2 cells were seeded in culture plates at $90 \%$ confluence. On the next day, the cells were treated with Tac $(60 \mu \mathrm{g} / \mathrm{ml})$ in the absence or presence of $1 \mu \mathrm{g} / \mathrm{ml}$ rKlotho and $25 \mu \mathrm{M} \mathrm{LY} 294002$ (LY, PI3K inhibitor). After a 12-h treatment, the cells were incubated in a non-CO ${ }_{2}$ incubator for $1 \mathrm{~h}$. Next, the ATP synthase inhibitor oligomycin, the uncoupler FCCP, or the respiratory chain complex I and III inhibitor rotenone/antimycin A were added to the culture medium, as indicated (a). The areas under the curve for basal respiration (b), ATP production (c), maximal respiration (d), and spare respiratory capacity (e) were calculated from the OCR. The data are presented as the mean \pm S.E. ${ }^{\#} P<0.05$ versus the VH group; ${ }^{\$} P<0.05$ versus the rKlotho groups; ${ }^{\circledR} P<0.05$ versus the Tac group; ! $P<0.05$ versus the Tac+rKlotho group

slide-mounted sections were heated for 60 min at $60^{\circ} \mathrm{C}$ in a HybEZTM hybridization oven (Advanced Cell Diagnostics). Tissues were dewaxed in xylene followed by dehydration in an ethanol series and air dried for 5 min. Tissue sections were incubated with pretreatment 1 solution (endogenous peroxidase block) for $10 \mathrm{~min}$ at RT. Slides were rinsed by immersion in double-distilled water ( $\mathrm{ddH} 2 \mathrm{O})$, followed by immersion in pretreatment 2 solution (antigen-retrieval citrate buffer) for $15 \mathrm{~min}$ at $100-104^{\circ} \mathrm{C}$. Slides were washed in $\mathrm{ddH} 2 \mathrm{O}$ and pretreatment 3 (protease) was applied for $30 \mathrm{~min}$ at $40^{\circ} \mathrm{C}$. Slides were washed in $\mathrm{ddH} 2 \mathrm{O}$, and target or control probes were incubated at $40^{\circ} \mathrm{C}$ for $2 \mathrm{~h}$ followed by rinsing in wash buffer (Advanced Cell Diagnostics) for 2 min at RT. Signal-amplification reagents 1-6 were applied sequentially for $30 \mathrm{~min}, 15 \mathrm{~min}, 30 \mathrm{~min}, 15 \mathrm{~min}, 30 \mathrm{~min}$, and $15 \mathrm{~min}$, respectively. Slides were rinsed in wash buffer for 2 min between amplification reagents. Incubations with amplifier reagents 1 to 4 were performed at $40^{\circ} \mathrm{C}$, whereas incubations with amplifier reagents 5 and 6 were performed at RT. Positive signals were visualized using DAB (Vector Laboratories). After drying for $15 \mathrm{~min}$ at $60^{\circ} \mathrm{C}$, slides were coverslipped using mounting media (Fisher Scientific, Medford, MA, USA). The positive control probe consisted of a proprietary probe for Bos taurus cyclophilin B, whereas the negative control probe targeted dapB of Bacillus subtilis.

Detection of 8-OHdG in urine. The end product of oxidative DNA damage was evaluated based on the level of the DNA adduct 8-OHdG in 24-h urine. The 8-OHdG concentration in urine was measured using a competitive ELISA (Cell Biolabs, San Diego, CA, USA).

Electron microscopy. After fixation in $2.5 \%$ glutaraldehyde in $0.1 \mathrm{M}$ phosphate buffer, renal cortex tissues were post-fixed with $1 \% \quad \mathrm{O}_{5} \mathrm{O}_{4}$ and embedded in Epon 812. Ultrathin sections were cut, stained with uranyl acetate/lead citrate, and photographed with a JEM-1200EX transmission electron microscope (JEOL Ltd., Tokyo, Japan). Sections were scanned randomly at 20 different spots per sample at $\times 5000$ magnification. The numbers, areas, and sizes of mitochondria were measured in 20 random proximal tubular cells, using imaging software (TDI Scope Eye). 
a

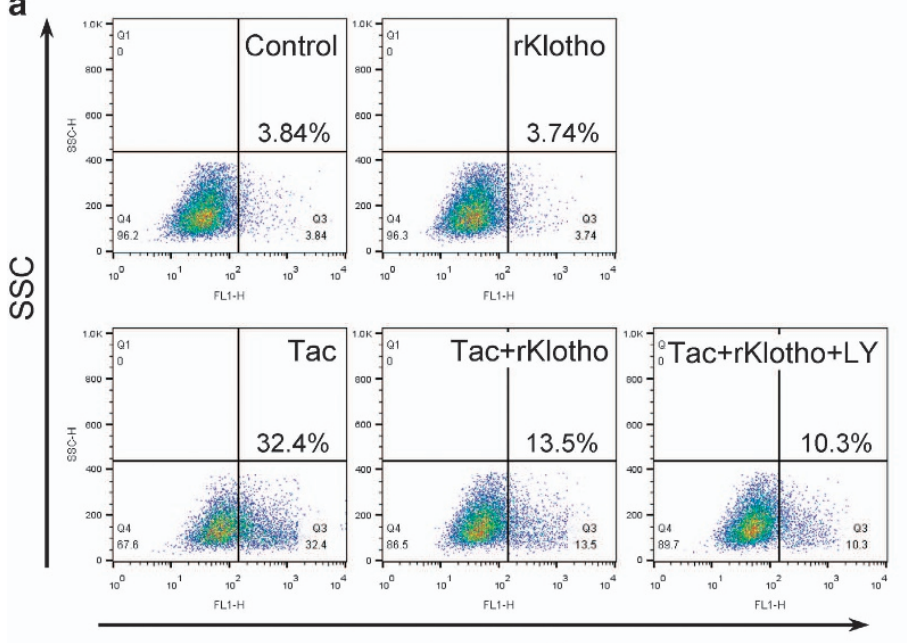

Annexin V

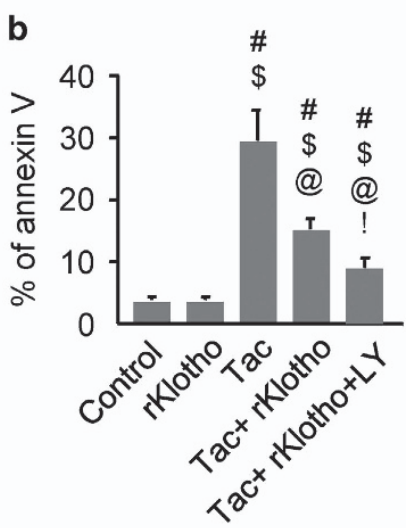

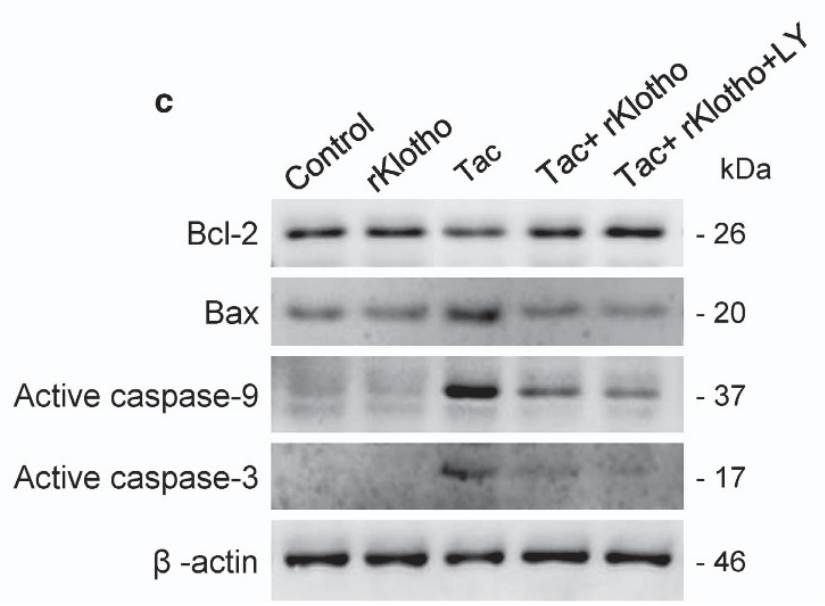

Figure 10 Klotho reduces Tac-induced apoptosis in HK-2 cells. HK-2 cells were seeded in culture plates at $90 \%$ confluence. On next day, the cells were treated with Tac $(60 \mu \mathrm{g} / \mathrm{ml})$ in the absence or presence of $1 \mu \mathrm{g} / \mathrm{ml}$ rKlotho and $25 \mu \mathrm{M} \mathrm{LY} 294002$ (LY, PI3K inhibitor) for $12 \mathrm{~h}$. Before the end of the treatment, annexin V solution was added to each well for $15 \mathrm{~min}$ to measure apoptosis, which was analyzed by flow cytometry. Whole-cell lysates were collected after each 12-h drug treatment to measure the relative protein expression levels of Bcl-2, Bax, active caspase- 9 and active caspase-3. (a and b) Flow cytometry histograms and a graph of annexin $\mathrm{V}$ labeling. (c) Representative western blots for Bcl-2, Bax, active caspase-9, and active caspase-3. All proteins are normalized to $\beta$-actin expression. LY; LY294002. The data are presented as the mean \pm S.E. ${ }^{\#} P<0.05$ versus the VH group; ${ }^{\$} P<0.05$ versus the rKlotho groups; ${ }^{\circledR} P<0.05$ versus the Tac group; ${ }^{P} P<0.05$ versus the Tac+rKlotho group

Cell culture. HK-2 cells from an immortalized human proximal tubular epithelial cell line were grown in Dulbecco's modified Eagle's medium (DMEM) containing $10 \%$ fetal bovine serum supplemented with $100 \mathrm{U} / \mathrm{ml}$ penicillin and $100 \mu \mathrm{g} / \mathrm{ml}$ streptomycin, and incubated at $37^{\circ} \mathrm{C}$ in a humidified atmosphere containing $5 \%$ $\mathrm{CO}_{2}$. The cells were seeded in culture plates and treated with Tac $(60 \mu \mathrm{g} / \mathrm{ml})$ and recombinant human Klotho (rKlotho; $1 \mu \mathrm{g} / \mathrm{ml}$, R\&D System), with or without LY294002 (25 $\mu \mathrm{M}$, Sigma-Aldrich) for $12 \mathrm{~h}$.

Cell-viability assay. Cells were seeded in 96-well plates at a density of $2 \times 10^{4}$ cells/well for $24 \mathrm{~h}$ and then subjected to various treatments for the specified periods. Before the end of the treatments, Cell Counting Kit (CCK)-8 solution (Dojindo Molecular Technologies, Kumamoto, Japan) was added to each well for $2 \mathrm{~h}$. Absorbance was measured at $450 \mathrm{~nm}$ using a VersaMax ELISA Reader (Molecular Devices, Sunnyvale, CA, USA).

Immunoblot analysis. Whole cells were lysed in PRO-PREP proteinextraction solution (Intron Biotechnology, Seongnam-si, Korea) according to the manufacturer's instructions. Equal amounts of protein were subjected to immunoblotting analysis with primary antibodies. Signals were detected using an enhanced chemiluminescence system (ATTO Corp., Tokyo, Japan). Quantification of relative densities was performed with the control group set at 100\%; densities were normalized to that of $\beta$-actin bands from the same gel (Quantity One version 4.4.0; Bio-Rad, Hercules, CA, USA).

Mitochondrial fractionation. Cells were harvested using a scraper and centrifuged at $500 \times g$. The cell pellet was washed by resuspension in phosphatebuffered saline. Mitochondria were separated by using the Mitochondria Isolation Kit (Pierce Biotechnology, Rockford, IL, USA). Mitochondria fractions were confirmed using a COX-IV antibody (A301-899A; Bethyl Laboratories).

ChIP experiments. ChIP was performed using a commercial kit (Millipore Corporation). In brief, cells were incubated with or without the rKlotho protein for $12 \mathrm{~h}$ during Tac treatment, after which they were cross-linked with $1 \%$ formaldehyde. After washing, the cells were sonicated and immunoprecipitated with an anti-FoxO3a antibody (ab12162; Abcam) overnight at $4{ }^{\circ} \mathrm{C}$. After elution and reverse cross-linking the antibody/DNA complexes, the cellular DNA was purified using a DNA purification kit (Qiagen, Hilden, Germany) and analyzed by quantitative real-time PCR (q-PCR) using primer pairs covering the MnSOD promoter region containing a FoxO3a-binding element at position -1249 (5'-GAGTATCTATAACCTGG TCCCAGCC-3' and 5'-GCTGAACCGTTTCCGTTGCTTCTTGC-3'). Data were shown as the amount of DNA relative to input. 


\section{Tac+rKlotho}

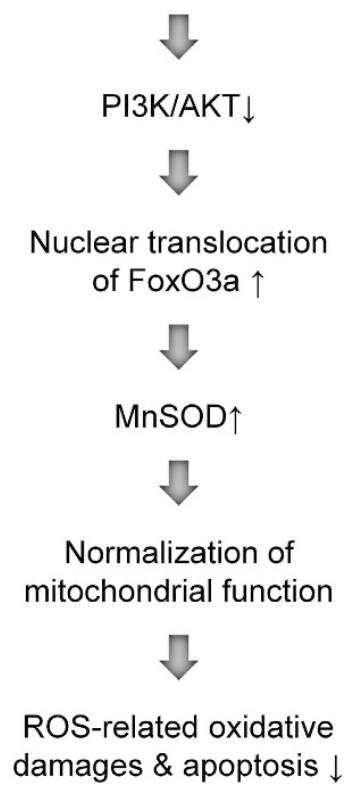

Normalization of mitochondrial function

\section{ROS-related oxidative damages \& apoptosis $\downarrow$}

Figure 11 Proposed mechanism of the protective effect of Klotho during Tac-induced renal injury

qRT-PCR. Total RNA from cultured cells were isolated using RNA-spin Total RNA Extraction Kit (Intron Biotechnology, Seongnam-si, Gyeonggi-do). First-strand cDNA was synthesized and subjected to q-PCR using SYBR Green Master Mix in a LightCycler 480 system (Roche, Rotkreuz, Switzerland). Gene expression was normalized to cyclophilin A expression using the change-in-threshold method and primers with the following sequences: $5^{\prime}$-GGTCCCAAAGACAGCAGAAA- $3^{\prime}$ and 5'-GTCACCACCCTGACACATAAA-3'.

Mitochondrial ROS detection. After treatment with the drug or $\mathrm{VH}$ mitochondrial ROS (superoxide anion) were detected using MitoSox Red (Invitrogen, Carlsbad, CA, USA) for $30 \mathrm{~min}$ at $37^{\circ} \mathrm{C}$ according to the manufacturer's instructions and analyzed using a FACS Calibur flow cytometer (BD Biosciences, San Jose, CA, USA) or an LSM700 confocal microscope (Carl Zeiss Microscopy $\mathrm{GmbH}$ ). Forward and side scatter data were collected (10 000 events per sample).

Determination of mitochondrial damage. Mitochondrial integrity was assessed using MitoTracker Deep Red FM (Invitrogen). After treatment with the drug or $\mathrm{VH}$ for $20 \mathrm{~min}$ at $37^{\circ} \mathrm{C}$ per the manufacturer's instructions, cells were analyzed using a FACS Calibur flow cytometer or an LSM700 confocal microscope.

Mitochondrial membrane potential $(\Delta \Psi \mathrm{m})$. The $\Delta \Psi \mathrm{m}$ of HK-2 cells was measured using the fluorescent, lipophilic cationic probe, JC-1 (Cayman, Ann Arbor, MI, USA). When live cells are incubated with JC-1, the dye penetrates the plasma membrane of cells as monomers and $\mathrm{JC}-1$ uptake into mitochondria is driven by the $\Delta \Psi \mathrm{m}$. Functional mitochondria are polarized and $\mathrm{JC}-1$ is rapidly taken up. This uptake raises the $\mathrm{JC}-1$ concentration leading to the formation of aggregates (J-aggregates) within mitochondria. Excited by a $488 \mathrm{~nm}$ laser, J-aggregates provoke a red spectral shift emission $(590 \mathrm{~nm})$. JC-1 does not accumulate in depolarized mitochondria, but instead remains in the cytoplasm as monomers, which emit green fluorescence $(525 \mathrm{~nm})$. Thus, in healthy cells, JC-1 fluorescence is seen in both the green (FL-1) and red (FL-2) channels, and a loss of red fluorescence indicates depolarized mitochondria. After treatment with the drug or $\mathrm{VH}$, the cells were incubated with $\mathrm{JC}-1$ staining solution for $20 \mathrm{~min}$ at $37^{\circ} \mathrm{C}$ according to the manufacturer's instructions and then analyzed using a FACS Calibur flow cytometer or an LSM700 confocal microscope.

OCR experiments. The cellular OCR rate was assessed in real-time with an XF24 Extracellular Flux Analyzer (Seahorse Biosciences, Billerica, MA, USA). After treatment with the drug or $\mathrm{VH}$, the cell medium was changed to running medium (DMEM supplemented with $5.5 \mathrm{mM}$ glucose, $1 \mathrm{mM}$ sodium pyruvate, $4 \mathrm{mM} \mathrm{L-}$ glutamine, $\mathrm{pH}$ 7.4) and incubated at $37^{\circ} \mathrm{C}$ in a non- $\mathrm{CO}_{2}$ incubator for $1 \mathrm{~h}$. The mitochondrial inhibitors used were the ATP synthase inhibitor oligomycin $(1 \mu \mathrm{M})$, carbonyl cyanide-4 (trifluoromethoxy) phenylhydrazone (FCCP; $0.5 \mu \mathrm{M}$ ), and the complex I and III inhibitor rotenone/antimycin A $(0.5 \mu \mathrm{M})$. OCR values were then normalized to the protein content of each sample. Mitochondrial function parameters were determined using these mitochondrial inhibitor compounds as modulators to determine several bioenergetics and mitochondrial function parameters, including basal respiration, ATP production, maximal respiration, and spare respiratory capacity, as described by Dott et al. ${ }^{37}$ Three to four wells were used for each experimental group.

Apoptosis. After treatment with the drug or $\mathrm{VH}$, trypsinized cells were incubated with $5 \mu \mathrm{l}$ of fluorescein isothiocyanate-conjugated annexin V (BD Biosciences) in $1 \times$ binding buffer (BD Biosciences) for $15 \mathrm{~min}$ at $\mathrm{RT}$, according to the manufacturer's protocol. The stained cells were analyzed by flow cytometry on a FACS Calibur instrument. Values are expressed as the percentage of fluorescent cells relative to the total cell count.

Statistical analysis. The data are expressed as the mean \pm standard error (S.E.) of at least three independent experiments. Multiple comparisons between groups were performed by one-way analysis of variance with Bonferroni's post hoc test, using SPSS software (Version 19.0; IBM Corp., Armonk, NY, USA). Results with $P$-values $<0.05$ were considered significant.

\section{Conflict of Interest}

The authors declare no conflict of interest.

Acknowledgements. This work has been supported by the Korean Health Technology R\&D Project, Ministry for Health \& Welfare, Republic of Korea (HI14C3417, HI16C1641), and Basic Science Research Program through the National Research Foundation of Korea (NRF) funded by the Ministry of Science, ICT \& Future Planning (NRF-2015R1A1A3A04000946).

\section{Publisher's Note}

Springer Nature remains neutral with regard to jurisdictional claims in published maps and institutional affiliations.

1. Kuro-o M, Matsumura $\mathrm{Y}$, Aizawa $\mathrm{H}$, Kawaguchi $\mathrm{H}$, Suga $\mathrm{T}$, Utsugi T et al. Mutation of the mouse klotho gene leads to a syndrome resembling ageing. Nature 1997; 390: 45-51.

2. Kurosu H, Yamamoto M, Clark J, Pastor J, Nandi A, Gurnani P et al. Suppression of aging in mice by the hormone Klotho. Science 2005; 309: 1829-1833.

3. Masuda H, Chikuda H, Suga T, Kawaguchi H, Kuro-o M. Regulation of multiple ageing-like phenotypes by inducible klotho gene expression in klotho mutant mice. Mecha Ageing Dev 2005; 126: 1274-1283.

4. Arking DE, Krebsova A, Macek M Sr., Macek M Jr., Arking A, Mian IS et al. Association of human aging with a functional variant of klotho. Proc Natl Acad Sci USA 2002; 99: 856-861.

5. Yamamoto M, Clark J, Pastor J, Gurnani P, Nandi A, Kurosu $\mathrm{H}$ et al. Regulation of oxidative stress by the anti-aging hormone klotho. J Biol Chem 2005; 280: 38029-38034.

6. Koh N, Fujimori T, Nishiguchi S, Tamori A, Shiomi S, Nakatani T et al. Severely reduced production of klotho in human chronic renal failure kidney. Biochem Biophys Res Commun 2001; 280: 1015-1020.

7. Sugiura H, Yoshida T, Tsuchiya K, Mitobe M, Nishimura S, Shirota S et al. Klotho reduces apoptosis in experimental ischaemic acute renal failure. Nephrol Dial Transplant 2005; 20: 2636-2645.

8. Haruna Y, Kashihara N, Satoh M, Tomita N, Namikoshi T, Sasaki T et al. Amelioration of progressive renal injury by genetic manipulation of Klotho gene. Proc Natl Acad Sci USA 2007; 104: 2331-2336.

9. Zhang X, Yalcin S, Lee D-F, Yeh T-Y, Lee S-M, Su J et al. FOXO1 is an essential regulator of pluripotency in human embryonic stem cells. Nat Cell Biol 2011; 13: 1092-1099.

10. Tzivion G, Dobson M, Ramakrishnan G. FoxO transcription factors; Regulation by AKT and 14-3-3 proteins. Biochim Biophys Acta 2011; 1813: 1938-1945.

11. Unger R. Klotho-induced insulin resistance: a blessing in disguise? Nat Med 2006; 12 : 56-57.

12. Emerling B, Weinberg F, Liu J-L, Mak T, Chandel N. PTEN regulates p300-dependent hypoxia-inducible factor 1 transcriptional activity through Forkhead transcription factor $3 \mathrm{a}$ (FOXO3a). Proc Natl Acad Sci USA 2008; 105: 2622-2627. 
13. Andreoni KA, Brayman KL, Guidinger MK, Sommers CM, Sung RS. Kidney and pancreas transplantation in the United States, 1996-2005. Am J Transplant 2007; 7: 1359-1375.

14. Takahashi N, Hayano T, Suzuki M. Peptidyl-prolyl cis-trans isomerase is the cyclosporin A-binding protein cyclophilin. Nature 1989; 337: 473-475.

15. Fischer G, Wittmann-Liebold B, Lang K, Kiefhaber T, Schmid FX. Cyclophilin and peptidylprolyl cis-trans isomerase are probably identical proteins. Nature 1989; 337: 476-478.

16. Harding MW, Galat A, Uehling DE, Schreiber SL. A receptor for the immunosuppressant FK506 is a cis-trans peptidyl-prolyl isomerase. Nature 1989; 341: 758-760.

17. Siekierka JJ, Hung SH, Poe M, Lin CS, Sigal NH. A cytosolic binding protein for the immunosuppressant FK506 has peptidyl-prolyl isomerase activity but is distinct from cyclophilin. Nature 1989; 341: 755-757.

18. Flanagan WM, Corthesy B, Bram RJ, Crabtree GR. Nuclear association of a T-cell transcription factor blocked by FK-506 and cyclosporin A. Nature 1991; 352: 803-807.

19. Jain J, McCaffrey PG, Miner Z, Kerppola TK, Lambert JN, Verdine GL et al. The T-cell transcription factor NFATp is a substrate for calcineurin and interacts with Fos and Jun. Nature 1993; 365: 352-355.

20. Shaw KT, Ho AM, Raghavan A, Kim J, Jain J, Park J et al. Immunosuppressive drugs prevent a rapid dephosphorylation of transcription factor NFAT1 in stimulated immune cells. Proc Natl Acad Sci USA 1995; 92: 11205-11209.

21. Clipstone NA, Crabtree GR. Identification of calcineurin as a key signalling enzyme in T-lymphocyte activation. Nature 1992; 357: 695-697.

22. O'Keefe SJ, Tamura J, Kincaid RL, Tocci MJ, O'Neill EA. FK-506- and CsA-sensitive activation of the interleukin-2 promoter by calcineurin. Nature 1992; 357: 692-694.

23. Emmel EA, Verweij CL, Durand DB, Higgins KM, Lacy E, Crabtree GR. Cyclosporin A specifically inhibits function of nuclear proteins involved in T cell activation. Science 1989; 246: $1617-1620$

24. Yoon HE, Yang CW. Established and newly proposed mechanisms of chronic cyclosporine nephropathy. Korean J Intern Med 2009; 24: 81-92.

25. Piao S, Kang S, Lim S, Chung B, Doh K, Heo S et al. Influence of N-acetylcysteine on Klotho expression and its signaling pathway in experimental model of chronic cyclosporine nephropathy in mice. Transplantation 2013; 96: 146-153.

26. Yoon HE, Choi BS. The renin-angiotensin system and aging in the kidney. Korean J Intern Med 2014; 29: 291-295.

27. Yoon HE, Lim SW, Piao SG, Song JH, Kim J, Yang CW. Statin upregulates the expression of klotho, an anti-aging gene, in experimental cyclosporine nephropathy. Nephron Exp Nephrol 2012; 120: e123-e133.

28. Yoon HE, Ghee JY, Piao S, Song JH, Han DH, Kim S et al. Angiotensin II blockade upregulates the expression of Klotho, the anti-ageing gene, in an experimental model of chronic cyclosporine nephropathy. Nephrol Dial Transplant 2011; 26: 800-813.
29. Jin J, Jin L, Lim S, Yang C. Klotho deficiency aggravates tacrolimus-induced renal Injury via the phosphatidylinositol 3-kinase-Akt-forkhead box potein O pathway. Am J Nephrol 2016; 43: 357-365.

30. Zorov D. Amelioration of aminoglycoside nephrotoxicity requires protection of renal mitochondria. Kidney Int 2010; 77: 841-843.

31. Rakugi H, Matsukawa N, Ishikawa K, Yang J, Imai M, Ikushima M et al. Anti-oxidative effect of Klotho on endothelial cells through cAMP activation. Endocrine 2007; 31: 82-87.

32. Schriner S, Linford N, Martin G, Treuting P, Ogburn C, Emond M et al. Extension of murine life span by overexpression of catalase targeted to mitochondria. Science 2005; 308: 1909-1911.

33. Abraham CR, Chen C, Cuny GD, Glicksman MA, Zeldich E. Small-molecule Klotho enhancers as novel treatment of neurodegeneration. Future Med Chem 2012; 4: 1671-1679.

34. King GD, Chen C, Huang MM, Zeldich E, Brazee PL, Schuman ER et al. Identification of novel small molecules that elevate Klotho expression. Biochem J 2012; 441: 453-461.

35. Zeldich E, Chen CD, Colvin TA, Bove-Fenderson EA, Liang J, Tucker Zhou TB et al. The neuroprotective effect of Klotho is mediated via regulation of members of the redox system. J Biol Chem 2014; 289: 24700-24715.

36. Lim S, Jin L, Piao S, Chung B, Yang C. Inhibition of dipeptidyl peptidase IV protects tacrolimus-induced kidney injury. Lab Invest 2015; 95: 1174-1185.

37. Dott W, Mistry P, Wright J, Cain K, Herbert KE. Modulation of mitochondrial bioenergetics in a skeletal muscle cell line model of mitochondrial toxicity. Redox Biol 2014; 2: 224-233.

(i) Cell Death and Disease is an open-access journal published by Nature Publishing Group. This work is licensed under a Creative Commons Attribution 4.0 International License. The images or other third party material in this article are included in the article's Creative Commons license, unless indicated otherwise in the credit line; if the material is not included under the Creative Commons license, users will need to obtain permission from the license holder to reproduce the material. To view a copy of this license, visit http://creativecommons.org/licenses/by/4.0/

(C) The Author(s) 2017 